\title{
Extracting many-body correlators of saturated gluons with precision from inclusive photon + dijet final states in deeply inelastic scattering
}

\author{
Kaushik Roy $\oplus^{1,2, *}$ and Raju Venugopalan ${ }^{2, \dagger}$ \\ ${ }^{1}$ Department of Physics and Astronomy, Stony Brook University, Stony Brook, New York 11794, USA \\ ${ }^{2}$ Physics Department, Brookhaven National Laboratory, Bldg. 510A, Upton, New York 11973, USA
}

(Received 2 December 2019; accepted 14 April 2020; published 29 April 2020)

\begin{abstract}
We highlight the principal results of a computation [1] in the color glass condensate effective field theory of the next-to-leading order (NLO) impact factor for inclusive photon + dijet production at Bjorken $x_{\mathrm{Bj}} \ll$ 1 in deeply inelastic electron-nucleus (e + A DIS) collisions. When combined with extant results for nextto-leading $\log x_{\mathrm{Bj}}$ JIMWLK renormalization group (RG) evolution of gauge invariant two-point (“dipole") and four-point ("quadrupole") correlators of lightlike Wilson lines, the inclusive photon + dijet $\mathrm{e}+\mathrm{A}$ DIS cross section can be determined to $\sim 10 \%$ accuracy. Our computation simultaneously provides the ingredients to compute fully inclusive DIS, inclusive photon, inclusive dijet, and inclusive photon + jet channels to the same accuracy. This makes feasible quantitative extraction of many-body correlators of saturated gluons and precise determination of the saturation scale $Q_{S, A}\left(x_{\mathrm{Bj}}\right)$ at a future Electron-Ion Collider. An interesting feature of our NLO result is the structure of the violation of the soft gluon theorem in the Regge limit. Another is the appearance in gluon emission of timelike nonglobal logs which also satisfy JIMWLK RG evolution.
\end{abstract}

DOI: 10.1103/PhysRevD.101.071505

The many-body recombination and screening of gluons in the high energy or small Bjorken $x_{\mathrm{Bj}}$ Regge limit of QCD competes with their rapid bremsstrahlung and leads to the perturbative unitarization of cross sections. This gluon saturation phenomenon $[2,3]$, in the color glass condensate (CGC) effective field theory (EFT) picture [4-10], occurs when the phase-space occupancy $n$ of gluons for transverse momenta $k_{\perp} \leq Q_{S}\left(x_{\mathrm{Bj}}\right)$ becomes of the order of the inverse of the QCD coupling $\alpha_{S}$. The saturation scale $Q_{S}\left(x_{\mathrm{Bj}}\right)$ is an emergent quantity and is the only large scale in the Regge limit; because it is much larger than intrinsic QCD scales, asymptotic freedom dictates that $\alpha_{S}\left(Q_{S}\right) \ll 1$. The large mode occupancy $n \sim 1 / \alpha_{S}\left(Q_{S}\right) \gg 1$ therefore suggests that gluon saturation corresponds to a remarkable classicalization of QCD at high energies.

In this paper, we will discuss the principal results of a first computation in the CGC EFT of the next-to-leading order (NLO) "impact factor" for inclusive photon + dijet production in deeply inelastic scattering of electrons off nuclei (e + ADIS) at high energies. The details of the computation are spelled out in a companion paper [1]. A

\footnotetext{
*kaushik.roy.1@ stonybrook.edu †raju@bnl.gov
}

Published by the American Physical Society under the terms of the Creative Commons Attribution 4.0 International license. Further distribution of this work must maintain attribution to the author(s) and the published article's title, journal citation, and DOI. Funded by SCOAP ${ }^{3}$. powerful motivation to perform the computation is the prospect of such measurements [11] at a future ElectronIon Collider (EIC) [12,13]. As we will outline here, knowing the NLO impact factor enables us to compute the photon + dijet cross section in $\mathrm{e}+\mathrm{A}$ DIS to $\mathrm{O}\left(\alpha_{S}^{3} \ln \left(1 / x_{\mathrm{Bj}}\right)\right)$ corresponding to $\sim 10 \%$ accuracy at the energies and nuclear saturation scales accessible at an EIC. While the accuracy is of sufficiently high order in $\alpha_{S}$ for tests of gluon saturation in the CGC EFT framework, we note that the associated relative error also depends on the choice of scheme and scale in the computation, and must be quantified for definitive comparison of theory to experiment [14].

We note that prior DIS studies of saturation at small $x$ focused on the NLO impact factor for fully inclusive DIS [15-23], a noteworthy exception being the recent study of diffractive dijet and exclusive vector meson production by Boussarie et al. [24-26]. As we will discuss, our computation has several novel features relative to these studies. We will note here only the most prominent of these: the sensitivity, at the stated high order accuracy, to a manybody "quadrupole" correlator which, in addition to the "dipole" correlator extracted from inclusive and diffractive DIS, plays a fundamental role in the infrared dynamics of high energy QCD.

To elaborate on our discussion, it is instructive to first briefly consider our previous computation [27] in the CGC EFT of the leading order (LO) inclusive photon + dijet $(\gamma+q \bar{q}) \mathrm{e}+\mathrm{A}$ DIS cross section: 


$$
\frac{\mathrm{d}^{3} \sigma^{\mathrm{LO} ; \gamma+q \bar{q}+X}}{\mathrm{~d} x \mathrm{~d} Q^{2} \mathrm{~d}^{6} K_{\perp} \mathrm{d}^{3} \eta_{K}}=\frac{\alpha_{e m}^{2} q_{f}^{4} y^{2} N_{c}}{512 \pi^{5} Q^{2}} \frac{1}{(2 \pi)^{4}} \frac{1}{2} L^{\mu \nu} \tilde{X}_{\mu \nu}^{\mathrm{LO}} .
$$

Here, $\alpha_{e m}=e^{2} / 4 \pi$ is the electromagnetic fine structure constant, $y=q \cdot P_{N} / \tilde{l} \cdot P_{N}$ is the inelasticity, $Q^{2}=-q^{2}>0$ is the squared momentum transfer from the nucleus, and $\mathrm{d}^{6} K_{\perp} \mathrm{d}^{3} \eta_{K}$ collectively denotes the phase-space density of the final state quark, antiquark, and photon. Likewise, $L^{\mu \nu}$ is the lepton tensor, corresponding to the emission of a virtual photon with four-momentum $q^{\mu}$ by the electron [28]. Our focus here is on the scattering of the virtual photon on the nuclear target producing the $\gamma+q \bar{q}$ final state and other (phase-space integrated) $X$ particles, described by the LO hadron tensor,

$$
\tilde{X}_{\mu \nu}^{\mathrm{LO}}=\int\left[\mathcal{D} \rho_{A}\right] W_{\Lambda_{0}^{-}}\left[\rho_{A}\right] \hat{X}_{\mu \nu}^{\mathrm{LO}}\left[\rho_{A}\right] .
$$

In a Born-Oppenheimer separation of modes in the EFT, the $\rho_{A}$ are the large $x_{\mathrm{Bj}}$ static color sources in the nucleus; these correspond to light cone longitudinal momentum modes with $\Lambda^{-}<\Lambda_{0}^{-}$. The initial distribution of such modes at the scale $\Lambda_{0}^{-}$is given by the nonperturbative gauge invariant weight functional $W_{\Lambda_{0}^{-}}\left[\rho_{A}\right]$. The small $x_{\mathrm{Bj}}$ dynamical gluon fields interacting with the probe correspondingly have longitudinal momenta above $\Lambda_{0}^{-}$; the leading order classical gluon field is a "shock wave" solution of the Yang-Mills (YM) equations in the presence of the sources $\rho^{a}(\boldsymbol{x})=$ $\tilde{\rho}^{a}\left(\boldsymbol{x}_{\perp}\right) \delta\left(x^{-}\right)$[29] of $\mathrm{O}(1 / g)$.

The solution of the YM equations in the Lorenz gauge $\partial_{\mu} A^{\mu}=0$ [or equivalently light cone (LC) $A^{-}=0$ gauge] is given by

$$
\begin{aligned}
& A_{\mathrm{cl}}^{+}=\int \frac{\mathrm{d}^{2} z_{\perp}}{4 \pi} \ln \frac{1}{\left(x_{\perp}-z_{\perp}\right)^{2} \Lambda^{2}} \rho_{A}\left(x^{-}, z_{\perp}\right), \\
& A_{\mathrm{cl}}^{-}=0 ; \quad A_{\mathrm{cl}, \perp}=0,
\end{aligned}
$$

where $\Lambda$ is an infrared cutoff necessary to invert the Laplace equation $-\nabla_{\perp}^{2} A_{\mathrm{cl}}^{+}=g \rho_{A}$. This solution in Lorenz gauge is simply related to the solution in the LC gauge $\tilde{A}^{+}=0$, with $\tilde{A}_{\mathrm{cl}}^{-}=0$ and $\tilde{A}_{\mathrm{cl}}^{i}=\frac{i}{g} U \partial^{i} U^{\dagger}$, where the adjoint lightlike Wilson line

$U\left(\boldsymbol{x}_{\perp}\right)=P_{-}\left(\exp \left\{-i g \int_{-\infty}^{+\infty} \mathrm{d} z^{-} A_{\mathrm{cl}}^{+, a}\left(z^{-}, \boldsymbol{x}_{\perp}\right) T^{a}\right\}\right)$

is expressed in terms of the large $x$ static color source densities via Eq. (3). Note that $T^{a}, a=1, \ldots, 8$, are the generators of color $S U(3)$ in the adjoint representation. This $x^{-}$path ordered exponential and its fundamental representation counterpart $\tilde{U}\left(\boldsymbol{x}_{\perp}\right)$ (obtained by replacing $T^{a}$ with the Gell-Mann matrices $t^{a}$ ) efficiently resum all

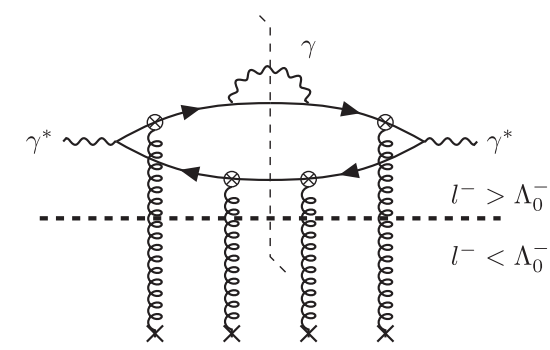

FIG. 1. A representative LO diagram. The cross-hatched open blobs represent the dressed quark propagator in $A^{-}=0$ gauge. See the text for details.

higher twist contributions $\frac{\rho_{A}}{\nabla_{\perp}^{2}} \rightarrow \frac{Q_{S}}{Q^{2}}$ from the multiple scattering of the $q \bar{q}$ pair off the color field of the nucleus.

The LO $\gamma+q \bar{q}$ amplitude is obtained by solving the Dirac equation in the $A_{\mathrm{cl}}^{+}$shock wave background in the $A^{-}=0 \mathrm{LC}$ gauge. A Feynman diagram for the $\mathrm{LO}$ process is shown in Fig. 1, where the vertical dashed line represents the cut separating the amplitude from its complex conjugate amplitude and the horizontal dashed line separates the dynamical projectile modes from the static target shock wave gauge fields at the scale $\Lambda_{0}^{-}$. The dressed shock wave propagator, denoted by cross-hatch circles in the figure, has the remarkably simple solution $[6,30]$

$$
S_{i j}(p, q)=S_{0}(p) \mathcal{T}_{q ; i j}(p, q) S_{0}(q),
$$

where $S_{0}(p)=\frac{i \not p}{p^{2}+i \varepsilon}$ is the free massless fermion propagator and

$$
\begin{aligned}
\mathcal{T}_{q ; j i}(q, p)= & 2 \pi \delta\left(p^{-}-q^{-}\right) \gamma^{-} \operatorname{sign}\left(q^{-}\right) \\
& \times \int \mathrm{d}^{2} z_{\perp} e^{-i\left(\boldsymbol{q}_{\perp}-\boldsymbol{p}_{\perp}\right) \cdot \boldsymbol{z}_{\perp}} \tilde{U}_{j i}^{\operatorname{sign}\left(q^{-}\right)}\left(\boldsymbol{z}_{\perp}\right)
\end{aligned}
$$

is the effective vertex corresponding to the multiple scattering of the quark or antiquark off the shock wave [31]. The LO computation of the $\gamma+q \bar{q}$ cross section in $A^{-}=0$ gauge is straightforward, and one finds

$$
\begin{aligned}
\tilde{X}_{\mu \nu}^{\mathrm{LO}}= & 2 \pi \delta\left(1-z_{q}-z_{\bar{q}}-z_{\gamma}\right) \int \mathrm{d} \Pi_{\perp}^{\mathrm{LO}} \int \mathrm{d} \Pi_{\perp}^{\prime \mathrm{LO} \star} \tau_{\mu \nu}^{q \bar{q}, q \bar{q}}\left(\boldsymbol{l}_{\perp}, \boldsymbol{l}_{\perp}^{\prime}\right) \\
& \times \Xi\left(\boldsymbol{x}_{\perp}, \boldsymbol{y}_{\perp} ; \boldsymbol{y}_{\perp}^{\prime}, \boldsymbol{x}^{\prime}{ }_{\perp}\right),
\end{aligned}
$$

where we introduced the compact notation [32],

$$
\int \mathrm{d} \Pi_{\perp}^{\mathrm{LO}}=\int_{\boldsymbol{l}_{\perp}} \int_{\boldsymbol{x}_{\perp}, \boldsymbol{y}_{\perp}} e^{i \boldsymbol{l}_{\perp}\left(\boldsymbol{x}_{\perp}-\boldsymbol{y}_{\perp}\right)-i\left(\boldsymbol{k}_{\perp}+\boldsymbol{k}_{\gamma_{\perp}}\right) \cdot \boldsymbol{x}_{\perp}-i p_{\perp} \cdot \boldsymbol{y}_{\perp}} .
$$

The function $\tau_{\mu \nu}^{q \bar{q}, q \bar{q}}\left(\boldsymbol{l}_{\perp}, \boldsymbol{l}_{\perp}{ }_{\perp}\right)$ denotes the spinor trace in the LO cross section [27].

The nonperturbative input from the dynamics of saturated gluons in the nuclear target is contained in 


$$
\Xi\left(\boldsymbol{x}_{\perp}, \boldsymbol{y}_{\perp} ; \boldsymbol{y}_{\perp}^{\prime}, \boldsymbol{x}_{\perp}^{\prime}\right)=1-D_{x y}-D_{y^{\prime} x^{\prime}}+Q_{y^{\prime} x^{\prime} ; x y} .
$$

Here,

$$
\begin{aligned}
D_{x y} & =\frac{1}{N_{c}}\left\langle\operatorname{Tr}\left(\tilde{U}\left(\boldsymbol{x}_{\perp}\right) \tilde{U}^{\dagger}\left(\boldsymbol{y}_{\perp}\right)\right)\right\rangle, \\
Q_{x y ; z w} & =\frac{1}{N_{c}}\left\langle\operatorname{Tr}\left(\tilde{U}\left(\boldsymbol{x}_{\perp}\right) \tilde{U}^{\dagger}\left(\boldsymbol{y}_{\perp}\right) \tilde{U}\left(z_{\perp}\right) \tilde{U}^{\dagger}\left(\boldsymbol{w}_{\perp}\right)\right)\right\rangle,
\end{aligned}
$$

represent respectively the dipole and quadrupole Wilson line correlators, where

$$
\langle\hat{\mathcal{O}}\rangle=\int\left[\mathcal{D} \rho_{A}\right] W_{\Lambda_{0}^{-}}\left[\rho_{A}\right] \hat{\mathcal{O}}\left[\rho_{A}\right]
$$

denotes the expectation value of a generic operator $\hat{\mathcal{O}}$. The weight functional $W_{\Lambda_{0}^{-}}\left[\rho_{A}\right]$ contains fundamental information about $n$-body correlations among the color sources at the scale $\Lambda_{0}^{-}$. In the McLerran-Venugopalan model (MV) [4-6] where it was introduced, $W_{\Lambda_{0}^{-}}\left[\rho_{A}\right]$ is Gaussian distributed for a large nucleus $[5,33,34]$ with a variance $\mu_{A}^{2} \sim A^{1 / 3}$, where $A$ denotes the atomic number. In the MV model, $\mu_{A}^{2} \propto Q_{S, 0}^{2}$, the saturation scale at $\Lambda_{0}^{-}[7,35]$. For quantitative estimates of the saturation scale at EIC energies, we refer the reader to Refs. $[13,36]$. In the CGC EFT, $D$ and $Q$ appear in a variety of LO processes in both $p+A$ and $e+A$ collisions [37].

We turn now to the extension of our computation of $\tilde{X}_{\mu \nu}$ to NLO, details of which are provided in Ref. [1]. Let us first consider the NLO diagram in Fig. 2. An important ingredient in our computation is the gluon "small fluctuations" propagator in the $A^{-}=0$ gauge classical shock wave background field [6,27,38-40],

$$
G_{\mu \nu ; a b}(p, q)=G_{\mu \rho ; a c}^{0}(p) \mathcal{T}_{g}^{\rho \sigma ; c d}(p, q) G_{\sigma \nu ; d b}^{0}(q),
$$

where $G_{\mu \rho ; a c}^{0}(p)=\frac{i}{p^{2}+i \varepsilon}\left(-g_{\mu \rho}+\frac{p_{\mu} n_{\rho}+n_{\mu} p_{\rho}}{n . p}\right) \delta_{a c}$ is the free propagator with Lorentz indices $\mu, \rho$; color indices $a, c$; and $n^{\mu}=\delta^{\mu+}$. The effective gluon vertex

$$
\begin{aligned}
\mathcal{T}_{g}^{\mu \nu ; a b}\left(p, p^{\prime}\right)= & -2 \pi \delta\left(p^{-}-p^{--}\right) \times\left(2 p^{-}\right) g^{\mu \nu} \operatorname{sign}\left(p^{-}\right) \\
& \left.\times \int \mathrm{d}^{2} z_{\perp} e^{-i\left(\boldsymbol{p}_{\perp}-\boldsymbol{p}_{\perp}^{\prime}\right)}\right) z_{\perp}\left(U^{a b}\right)^{\operatorname{sign}\left(p^{-}\right)}\left(\boldsymbol{z}_{\perp}\right),
\end{aligned}
$$

corresponding to multiple scattering of the gluon off the shock wave background field, is represented by the filled blobs in Fig. 2. Unlike many of the computations in the literature, the simple momentum space structure in Eqs. (5) and (12) allow us to carry out our higher order computations directly in momentum space.

In the NLO diagrams represented in Fig. 2, the contributions enhanced by $\alpha_{S} \ln \left(\Lambda_{1}^{-} / \Lambda_{0}^{-}\right)$[with $\Lambda_{1}^{-}$chosen

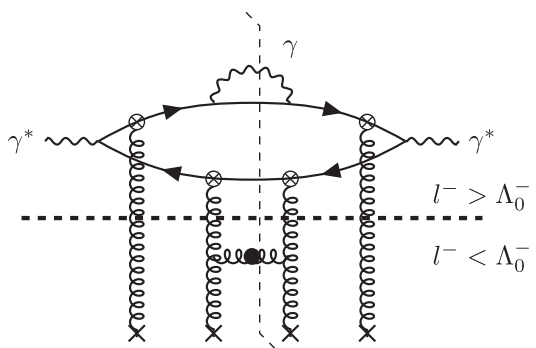

FIG. 2. NLO leading logs in $x\left(\alpha_{S} \ln (1 / x) \sim 1\right)$ diagram with the same $\mathrm{O}(1)$ contributions as the LO diagram in Fig. 1. The filled blob represents the dressed gluon propagator in $A^{-}=0$ gauge. See the text for details.

such that these terms are $\mathrm{O}(1)$ ] can be combined with the LO result in Eq. (2) and expressed as [41]

$$
\begin{aligned}
\tilde{X}_{\mu \nu}^{\mathrm{LO}}+\delta \tilde{X}_{\mu \nu}^{\mathrm{NLO}: 1}= & \int\left[\mathcal{D} \rho_{A}\right]\left(1+\ln \left(\Lambda_{1}^{-} / \Lambda_{0}^{-}\right) \mathcal{H}_{\mathrm{LO}}\right) W_{\Lambda_{0}^{-}}\left[\rho_{A}\right] \\
& \times \hat{X}_{\mu \nu}^{\mathrm{LO}}\left[\rho_{A}\right] .
\end{aligned}
$$

Further redefining

$$
\left(1+\ln \left(\Lambda_{1}^{-} / \Lambda_{0}^{-}\right) \mathcal{H}_{\mathrm{LO}}\right) W_{\Lambda_{0}^{-}}\left[\rho_{A}\right]=W_{\Lambda_{1}^{-}}\left[\rho_{A}\right]
$$

and thereby absorbing the semifast gluon fluctuations of the target in a modification of the weight functional of the color sources at the scale $\Lambda_{1}^{-}$, one obtains the leading log in $x$ (LL $x)$ [42] JIMWLK renormalization group (RG) equation [43],

$$
\frac{\partial}{\partial\left(\ln \Lambda^{-}\right)} W_{\Lambda^{-}}\left[\rho_{A}\right]=\mathcal{H}_{\mathrm{LO}} W_{\Lambda^{-}}\left[\rho_{A}\right]
$$

where $\mathcal{H}_{\mathrm{LO}}$ is the well-known JIMWLK Hamiltonian [44-47]. We will henceforth label the weight functional that satisfies Eq. (16) as $W^{L L x}\left[\rho_{A}\right]$.

At next-to-next-to-leading order (NNLO) in $\alpha_{S}$, there are two relevant classes of contributions as illustrated in Fig. 3.

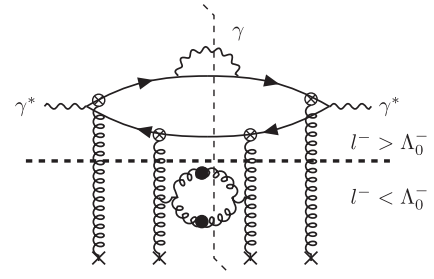

(a)

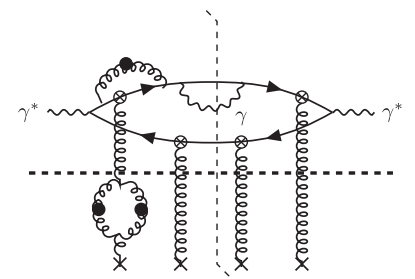

(b)
FIG. 3. (a) NNLO diagram corresponding to target fluctuations of $\mathrm{O}\left(\alpha_{S}^{2} \ln (1 / x)\right)$. Such contributions are resummed to all orders by the NLO JIMWLK equation. (b) NNLO diagram with $\mathrm{O}\left(\alpha_{S} \ln (1 / x)\right)$ fluctuations of the target and $\mathrm{O}\left(\alpha_{S}\right)$ fluctuations of the projectile. These projectile fluctuations constitute the NLO $\gamma+$ dijet impact factor. 
Diagrams corresponding to a two-loop fluctuation of the target are shown in Fig. 3(a). For such two-loop diagrams, contributions [48] of order $\alpha_{S}^{2} \ln ^{2}\left(\Lambda_{1}^{-} / \Lambda_{0}^{-}\right) \sim O(1)$ are included in $W^{L L x}\left[\rho_{A}\right]$. We will therefore consider here only the two-loop diagrams that contain next-to-leading logarithms in $x$ (NLL $x$ ) contributions to Eq. (14). The LO + NLL $x$ result including these can be expressed as

$$
\tilde{X}_{\mu \nu}^{\mathrm{LO}}+\delta \tilde{X}_{\mu \nu}^{\mathrm{NLL} x}=\int\left[\mathcal{D} \rho_{A}\right] W_{\Lambda_{1}^{-}}^{N L L x}\left[\rho_{A}\right] \hat{X}_{\mu \nu}^{\mathrm{LO}}\left[\rho_{A}\right],
$$

where

$W_{\Lambda_{1}^{-}}^{N L L x}\left[\rho_{A}\right]=\left\{1+\ln \left(\Lambda_{1}^{-} / \Lambda_{0}^{-}\right)\left(\mathcal{H}_{\mathrm{LO}}+\mathcal{H}_{\mathrm{NLO}}\right)\right\} W_{\Lambda_{0}^{-}}\left[\rho_{A}\right]$,

and the NLO JIMWLK Hamiltonian $\mathcal{H}_{\mathrm{NLO}}$ computed in Refs. [49-53] (see also Refs. [54,55]) is of order $\alpha_{S}^{2}$.

The second class of NNLO contributions [formally of $\mathrm{O}\left(\alpha_{S}^{2}\right)$ ], shown in Fig. 3(b), corresponds to one-loop fluctuations of both the projectile and the target. Specifically, the $\alpha_{S} \ln (1 / x)$ contributions from the gluon fluctuations below the cut $\Lambda_{0}^{-}$are matched to the finite contributions above the cut (without logarithms) of $\mathrm{O}\left(\alpha_{S}\right)$ in the real and virtual corrections to the LO photon + dijet projectile final state. These finite terms constitute the NLO $\gamma+$ dijet impact factor. Together, they give for the class of NNLO contributions in Fig. 3(b)

$$
\tilde{X}_{\mu \nu}^{\mathrm{NNLO} ; \text { finite }}=\int\left[\mathcal{D} \rho_{A}\right] W^{L L x}\left[\rho_{A}\right] \hat{X}_{\mu \nu}^{\mathrm{NLO} ; \text { finite }}\left[\rho_{A}\right] .
$$

Combining the expressions in Eqs. (17) and (19), the hadron tensor for inclusive photon + dijet production to $\mathrm{NLO}+$ NLL $x$ accuracy can be written as

$$
\begin{aligned}
\tilde{X}_{\mu \nu}^{\mathrm{NLO}+\mathrm{NLLx}} & =\int\left[\mathcal{D} \rho_{A}\right]\left\{W^{N L L x}\left[\rho_{A}\right] \hat{X}_{\mu \nu}^{\mathrm{LO}}\left[\rho_{A}\right]\right. \\
& \left.+W^{L L x}\left[\rho_{A}\right] \hat{X}_{\mu \nu}^{\mathrm{NLO} ; \text { finite }}\left[\rho_{A}\right]\right\} \\
& \simeq \int\left[\mathcal{D} \rho_{A}\right]\left(W^{N L L x}\left[\rho_{A}\right]\left\{\hat{X}_{\mu \nu}^{\mathrm{LO}}\left[\rho_{A}\right]+\hat{X}_{\mu \nu}^{\mathrm{NLO} ; \text { finite }}\left[\rho_{A}\right]\right\}\right. \\
& \left.+\mathrm{O}\left(\alpha_{S}^{3} \ln \left(\Lambda_{1}^{-} / \Lambda_{0}^{-}\right)\right)\right) .
\end{aligned}
$$

Our knowledge of the NLO impact factor and NLLx JIMWLK evolution can be combined, as shown above and in Fig. 4, to extend the scope of the computation to $O\left(\alpha_{S}^{3} \ln (1 / x)\right)$. However, as the $\simeq$ symbol indicates, this knowledge is insufficient to capture all the diagrams that contribute to this accuracy.

We shall now sketch the key features of the computation of the inclusive NLO photon + dijet impact factor in Ref. [1]. The quantum fluctuations (with $l^{-}>\Lambda_{0}^{-}$) that contribute toward the NLO impact factor can be broadly classified into

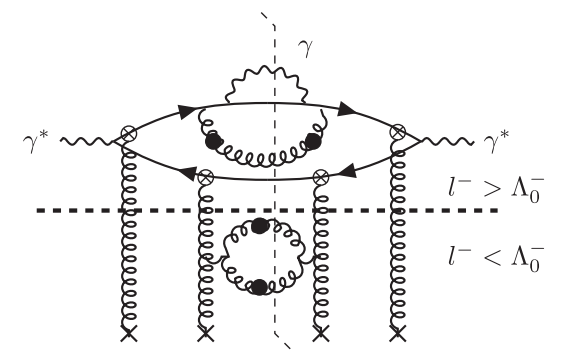

FIG. 4. $\mathrm{N}^{3} \mathrm{LO}$ diagrams including $\mathrm{O}\left(\alpha_{S}\right)$ fluctuations of the projectile and $\mathrm{O}\left(\alpha_{S}^{2} \ln (1 / x)\right)$ fluctuations of the target. Such contributions can be computed using extant results for NLO JIMWLK and the NLO impact factor computed in Ref. [1].

the modulus squared of real gluon emission amplitudes and the interference of virtual gluon exchange processes with LO diagrams. In each case, the real or virtual gluon can scatter off the shock wave or propagate freely without scattering; in addition, there are all possible permutations of the emission vertex of the final state photon. For real emissions alone, there are 400 possible diagrams - see Ref. [1] for the complete set of real and virtual graphs contributing at NLO. These can be categorized systematically by their color structures, allowing one to clearly observe the cancellation of the soft, collinear, and UV divergences that arise in the intermediate steps of our computation.

Soft singularities arise from the spurious $l^{-}=0$ pole in the free gluon propagator in $A^{-}=0 \mathrm{LC}$ gauge. These are regulated by imposing a cutoff at the initial scale of evolution $\Lambda_{0}^{-}$. We show in Ref. [1] that log divergent terms in $\Lambda_{0}^{-}$in the "slow" gluon $l^{-} \rightarrow 0$ limit possess color structures at NLO identical to those resulting from the action (as shown in Ref. [56]) of the JIMWLK kernel on $\Xi$ in Eq. (9). Our computation therefore provides an explicit proof of high energy JIMWLK factorization for a nontrivial process other than fully inclusive DIS.

The UV divergences are extracted using dimensional regularization in $d=2-\epsilon$ dimensions. For gluon loops, most of the UV divergences cancel between graphs at the amplitude level. There are, however, residual collinear divergences. Such collinear singularities also arise from real gluon emission when integrating over the phase space in which the gluon can be collinear to the quark or antiquark. Because we are not integrating over the momenta of our $\gamma+q \bar{q}$ final state, there are collinear divergences that survive the real-virtual cancellations. These can be absorbed into a jet algorithm.

IR safe quantities are obtained by promoting the partons to jets and working in the small cone approximation [57] of jet cone radius $R \ll 1$. This restricts the integration over the phase space for the real gluon. The dominant contribution is of the form $\alpha_{S}(A \ln (R)+B)$, where $A, B$, spelled out in Ref. [1], are of $\mathrm{O}(1)$; all noncollinearly divergent contributions are phase-space suppressed by powers of $R^{2}$. 
The jet algorithm also allows for a cancellation of softcollinear divergences between soft gluon emissions inside and outside the jet cone. In the latter case, we observe that slow gluon emissions at wide angles $\left(l^{-} \rightarrow 0\right.$ but any $\left.l_{\perp}\right)$ satisfy JIMWLK evolution and must be subtracted from the jet cross section to avoid double counting when the NLO impact factor is combined with small $x$ evolution [58]. This result is an explicit realization of the conformal spacelike-timelike correspondence previously conjectured by Mueller [59].

We also observe interestingly that, as a consequence of the different topologies of the color structures that contribute toward soft and collinear divergences, the soft gluon theorem is violated for inclusive photon + dijet production. This result is new and is unlike the case of diffractive DIS [25]. The factorization violating term has the color structure $(Q-D D)$ and has significant implications for the infrared structure of QCD in the Regge limit. In particular, the building block of $Q$ and $D$ is the $x^{-}$path ordered Wilson line in Eq. (4); it would therefore be interesting to explore if the soft gluon theorem is restored by modifying the boundary conditions of the quadrupole and dipole operators at $x^{-}= \pm \infty[60]$.

After due consideration of all divergences, our final result for the triple differential cross section for the $\gamma+q \bar{q}$ jet production in e + A DIS is

$$
\begin{aligned}
\frac{\mathrm{d}^{3} \sigma^{\mathrm{LO}+\mathrm{NLO}+\mathrm{NLL} x \text {;jet }}}{\mathrm{d} x \mathrm{~d} Q^{2} \mathrm{~d}^{6} K_{\perp} \mathrm{d}^{3} \eta_{K}}= & \frac{\alpha_{e m}^{2} q_{f}^{4} y^{2} N_{c}}{512 \pi^{5} Q^{2}} \frac{1}{(2 \pi)^{4}} \frac{1}{2} \\
& \times L^{\mu \nu} \tilde{X}_{\mu \nu}^{\mathrm{LO}+\mathrm{NLO}+\mathrm{NLL} x \text {;jet }},
\end{aligned}
$$

where the hadron tensor at $\mathrm{O}\left(\alpha_{S}^{3} \ln (1 / x)\right)$ accuracy can be written as

$\tilde{X}_{\mu \nu}^{\mathrm{LO}+\mathrm{NLO}+\mathrm{NLL} x ; \mathrm{jet}}=\int\left[\mathcal{D} \rho_{A}\right] W_{x_{\mathrm{Bj}}}^{N L L x}\left[\rho_{A}\right]\left[\left(1+\frac{2 \alpha_{S} C_{F}}{\pi}\left\{-\frac{3}{4} \ln \left(\frac{R^{2}\left|\boldsymbol{p}_{J \perp}\right|\left|\boldsymbol{p}_{K \perp}\right|}{4 z_{J} z_{K} Q^{2} e^{\gamma_{E}}}\right)+\frac{7}{4}-\frac{\pi^{2}}{6}\right\}\right) \tilde{X}_{\mu \nu}^{\mathrm{LO} ; \mathrm{jet}}\left[\rho_{A}\right]+\tilde{X}_{\mu \nu ; \text { finite }}^{\mathrm{NLO}}\left[\rho_{A}\right]\right]$.

In this expression [61], the finite terms $\tilde{X}_{\mu \nu \text {;inite }}^{\mathrm{NLO} \text {;inite }}$ are of order $\alpha_{S}$ relative to the leading term and constitute the NLO impact factor. The explicit results for these are the principal results of Ref. [1]. For the virtual gluon diagrams, where the isolation of divergent and finite pieces is done at the amplitude level, it is straightforward albeit tedious to derive analytical expressions for such terms. This is, however, not possible for real gluon emission graphs; we need to evaluate the finite pieces numerically. These are obtained by taking the modulus squared of the real emission amplitudes, integrating over the gluon phase space with a cutoff, implementing the jet algorithm, and subsequently subtracting the pieces that contribute to leading log JIMWLK evolution.

The numerical computation of the finite pieces constitut-

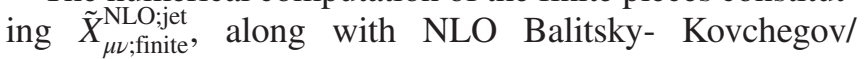
JIMWLK evolution, will provide the necessary ingredients to compute photon + dijet production (and associated measurement channels) in $\mathrm{e}+\mathrm{A}$ DIS to $\mathrm{O}\left(\alpha_{S}^{3} \ln (1 / x)\right)$ accuracy [62]. Carrying out this program for such nontrivial final states in the kinematics relevant for the EIC is of great importance for evaluating the discovery potential for gluon saturation. Specifically, unlike inclusive DIS, our NLO results are sensitive to both $D$ and $Q$ many-body correlators. A deeper understanding of saturation will require tests of the universality of these correlators in multiple $\mathrm{e}+\mathrm{A}$ channels and, ideally, in the $\mathrm{p}+$ A final states in which they also appear. This will require a global data-theory analysis analogous to the extraction of parton distribution functions in collinear perturbative QCD.

The simple forms of the momentum space shockwave propagators in $A^{-}=0$ gauge and the momentum space techniques employed in our work [1,27] allow us to extend fluctuations of the projectile to two loops. This is important for a systematic treatment of the running of the coupling both in the impact factor and in the NLO RG evolution equation and in establishing the precision of the computation with variations of scale and scheme. Finally, this framework can be applied in $\mathrm{p}+\mathrm{A}$ collisions beyond the current state of the art for inclusive hadron [63-67], quarkonium $[68,69]$, and photon production [70-72], to NNLO in the CGC power counting and beyond.

\section{ACKNOWLEDGMENTS}

This material is based on work supported by the U.S. Department of Energy, Office of Science, Office of Nuclear Physics, under Contract No. de-sc0012704 and within the framework of the TMD Theory Topical Collaboration. $\mathrm{K}$. R. is supported by an LDRD grant from Brookhaven Science Associates and by the Joint BNL-Stony Brook Center for Frontiers in Nuclear Science. 
[1] K. Roy and R. Venugopalan, NLO impact factor for inclusive photon + dijet production in e + A DIS at small $x$, Phys. Rev. D 101, 034028 (2020).

[2] L. V. Gribov, E. M. Levin, and M. G. Ryskin, Semihard processes in QCD, Phys. Rep. 100, 1 (1983).

[3] A.H. Mueller and J.-W. Qiu, Gluon recombination and shadowing at small values of $x$, Nucl. Phys. B268, 427 (1986).

[4] L. D. McLerran and R. Venugopalan, Computing quark and gluon distribution functions for very large nuclei, Phys. Rev. D 49, 2233 (1994).

[5] L. D. McLerran and R. Venugopalan, Gluon distribution functions for very large nuclei at small transverse momentum, Phys. Rev. D 49, 3352 (1994).

[6] L. D. McLerran and R. Venugopalan, Green's functions in the color field of a large nucleus, Phys. Rev. D 50, 2225 (1994).

[7] E. Iancu and R. Venugopalan, The Color glass condensate and high-energy scattering in QCD, in Quark Gluon Plasma, edited by R. C. Hwa et al. (World Scientific, Singapore, 2003), pp. 249-3363.

[8] F. Gelis, E. Iancu, J. Jalilian-Marian, and R. Venugopalan, The color glass condensate, Annu. Rev. Nucl. Part. Sci. 60, 463 (2010).

[9] Y. V. Kovchegov and E. Levin, Quantum Chromodynamics at High Energy (Cambridge University Press, Cambridge, England, 2012), Vol. 33.

[10] J.-P. Blaizot, High gluon densities in heavy ion collisions, Rep. Prog. Phys. 80, 032301 (2017).

[11] Our highly differential computation will allow us to provide, to the same accuracy, cross sections for inclusive dijet, inclusive photon, inclusive photon, and fully inclusive DIS measurements.

[12] A. Accardi et al., Electron ion collider: The next QCD frontier, Eur. Phys. J. A 52, 268 (2016).

[13] E. C. Aschenauer, S. Fazio, J. H. Lee, H. Mantysaari, B. S. Page, B. Schenke, T. Ullrich, R. Venugopalan, and P. Zurita, The electron-ion collider: Assessing the energy dependence of key measurements, arXiv:1708.01527.

[14] We will elaborate on this important point later in the paper.

[15] J. Bartels, S. Gieseke, and C.F. Qiao, The $\left(\gamma^{*} \rightarrow q \bar{q}\right)$ Reggeon vertex in next-to-leading order QCD, Phys. Rev. D 63, 056014 (2001); Erratum, 65, 079902 (2002).

[16] J. Bartels, D. Colferai, S. Gieseke, and A. Kyrieleis, NLO corrections to the photon impact factor: Combining real and virtual corrections, Phys. Rev. D 66, 094017 (2002).

[17] J. Bartels, S. Gieseke, and A. Kyrieleis, The process $\gamma^{*}(L)+q \rightarrow(q \bar{q} g)+q$ : Real corrections to the virtual photon impact factor, Phys. Rev. D 65, 014006 (2001).

[18] I. Balitsky and G. A. Chirilli, Photon impact factor in the next-to-leading order, Phys. Rev. D 83, 031502 (2011).

[19] I. Balitsky and G. A. Chirilli, Photon impact factor and $k_{T^{-}}$ factorization for DIS in the next-to-leading order, Phys. Rev. D 87, 014013 (2013).

[20] G. Beuf, NLO corrections for the dipole factorization of DIS structure functions at low x, Phys. Rev. D 85, 034039 (2012).

[21] G. Beuf, Dipole factorization for DIS at NLO: Loop correction to the $\gamma_{T, L}^{*} \rightarrow q \bar{q}$ light-front wave functions, Phys. Rev. D 94, 054016 (2016).
[22] G. Beuf, Dipole factorization for DIS at NLO: Combining the $q \bar{q}$ and $q \bar{q} g$ contributions, Phys. Rev. D 96, 074033 (2017).

[23] H. Hanninen, T. Lappi, and R. Paatelainen, One-loop corrections to light cone wave functions: The dipole picture DIS cross section, Ann. Phys. (Amsterdam) 393, 358 (2018).

[24] R. Boussarie, A. V. Grabovsky, L. Szymanowski, and S. Wallon, Impact factor for high-energy two and three jets diffractive production, J. High Energy Phys. 09 (2014) 026.

[25] R. Boussarie, A. V. Grabovsky, L. Szymanowski, and S. Wallon, On the one loop $\gamma^{(*)} \rightarrow q \bar{q}$ impact factor and the exclusive diffractive cross sections for the production of two or three jets, J. High Energy Phys. 11 (2016) 149.

[26] R. Boussarie, A. V. Grabovsky, D. Yu. Ivanov, L. Szymanowski, and S. Wallon, Next-to-Leading Order Computation of Exclusive Diffractive Light Vector Meson Production in a Saturation Framework, Phys. Rev. Lett. 119, 072002 (2017).

[27] K. Roy and R. Venugopalan, Inclusive prompt photon production in electron-nucleus scattering at small x, J. High Energy Phys. 05 (2018) 013.

[28] Explicit expressions for all kinematic variables are provided in Refs. [1,27].

[29] The delta function corresponds to the Lorentz contraction of the sources in a frame where the momentum of the right moving nucleus $P_{N}^{+} \rightarrow \infty$.

[30] L. D. McLerran and R. Venugopalan, Fock space distributions, structure functions, higher twists and small x, Phys. Rev. D 59, 094002 (1999).

[31] The 1 term in the expansion of the Wilson lines corresponds to the possibility that either the quark or the antiquark does not scatter. Since at least one of them must, one should subtract a term from the net amplitude wherein $\tilde{U}$ (and $U$ ) are set to unity everywhere.

[32] The second such term appearing in Eq. (7) results from the complex conjugate of Eq. (8) and corresponds to replacing all transverse coordinates and internal momenta therein by their primed counterparts.

[33] Y. V. Kovchegov, NonAbelian Weizsacker-Williams field and a two-dimensional effective color charge density for a very large nucleus, Phys. Rev. D 54, 5463 (1996).

[34] S. Jeon and R. Venugopalan, Random walks of partons in $\mathrm{SU}(\mathrm{N}(\mathrm{c}))$ and classical representations of color charges in QCD at small x, Phys. Rev. D 70, 105012 (2004).

[35] T. Lappi, Wilson line correlator in the MV model: Relating the glasma to deep inelastic scattering, Eur. Phys. J. C 55, 285 (2008).

[36] H. Kowalski, T. Lappi, and R. Venugopalan, Nuclear Enhancement of Universal Dynamics of High Parton Densities, Phys. Rev. Lett. 100, 022303 (2008).

[37] Group theory techniques to compute such correlators in the MV model are discussed in Refs. [73-76].

[38] A. Ayala, J. Jalilian-Marian, L. D. McLerran, and R. Venugopalan, Quantum corrections to the WeizsackerWilliams gluon distribution function at small $\mathrm{x}$, Phys. Rev. D 53, 458 (1996).

[39] A. Ayala, J. Jalilian-Marian, L. D. McLerran, and R. Venugopalan, The Gluon propagator in nonAbelian Weizsacker-Williams fields, Phys. Rev. D 52, 2935 (1995). 
[40] I. I. Balitsky and A. V. Belitsky, Nonlinear evolution in high density QCD, Nucl. Phys. B629, 290 (2002).

[41] We employ here the Hermiticity of $W$ with respect to the functional integration over $\rho_{A}$.

[42] The LC momentum fraction $x$ is equated to the Bjorken variable $x_{\mathrm{Bj}}$ here. The precise relation between the two is established beyond this order of the computation.

[43] To LL $x$, this equation generates the Balitsky hierarchy [77] describing the evolution of $\mathrm{n}$-point Wilson line correlators in $x$. In the limit of large number of colors $N_{c}$, and for $A \gg 1$, the simplest dipole correlator of lightlike Wilson lines in this hierarchy satisfies the Balitsky-Kovchegov equation [77,78]. In the leading twist limit where $Q_{S}^{2}(x) / Q^{2} \ll 1$, it reduces to the BFKL equation $[79,80]$.

[44] J. Jalilian-Marian, A. Kovner, A. Leonidov, and H. Weigert, The Wilson renormalization group for low $\mathrm{x}$ physics: Towards the high density regime, Phys. Rev. D 59, 014014 (1998).

[45] J. Jalilian-Marian, A. Kovner, and H. Weigert, The Wilson renormalization group for low x physics: Gluon evolution at finite parton density, Phys. Rev. D 59, 014015 (1998).

[46] E. Iancu, A. Leonidov, and L. D. McLerran, Nonlinear gluon evolution in the color glass condensate. 1., Nucl. Phys. A692, 583 (2001).

[47] E. Ferreiro, E. Iancu, A. Leonidov, and L. McLerran, Nonlinear gluon evolution in the color glass condensate. 2. Nucl. Phys. A703, 489 (2002).

[48] There are also contributions from two-loop QCD diagrams proportional to $\alpha_{S}^{2}$ alone (without leading logs in $x$ ), but these are suppressed at the desired accuracy of our problem.

[49] I. Balitsky and G. A. Chirilli, Rapidity evolution of Wilson lines at the next-to-leading order, Phys. Rev. D 88, 111501 (2013).

[50] A. Kovner, M. Lublinsky, and Y. Mulian, Jalilian-Marian, Iancu, McLerran, Weigert, Leonidov, Kovner evolution at next to leading order, Phys. Rev. D 89, 061704 (2014).

[51] I. Balitsky and A. V. Grabovsky, NLO evolution of 3-quark Wilson loop operator, J. High Energy Phys. 01 (2015) 009.

[52] M. Lublinsky and Y. Mulian, High energy QCD at NLO: from light-cone wave function to JIMWLK evolution, J. High Energy Phys. 05 (2017) 097.

[53] S. Caron-Huot, When does the gluon reggeize?, J. High Energy Phys. 05 (2015) 093.

[54] Y. V. Kovchegov and H. Weigert, Triumvirate of running couplings in small-x evolution, Nucl. Phys. A784, 188 (2007).

[55] M. A. Braun, Pomeron with a running coupling in the nucleus, Eur. Phys. J. C 51, 625 (2007).

[56] F. Dominguez, A. H. Mueller, S. Munier, and B.-W. Xiao, On the small- $x$ evolution of the color quadrupole and the Weizsäcker-Williams gluon distribution, Phys. Lett. B 705, 106 (2011).

[57] D. Yu. Ivanov and A. Papa, The next-to-leading order forward jet vertex in the small-cone approximation, J. High Energy Phys. 05 (2012) 086.

[58] This correspondence is a feature of nonglobal logarithms in jet physics [81]; the latter was identified with BalitskyKovchegov/JIMWLK evolution by Marchesini and Mueller [82] as well as by Weigert [83] and subsequently significantly developed by Hatta et al. [84-87] and by Neill [88].
[59] A.H. Mueller, Conformal spacelike-timelike correspondence in QCD, J. High Energy Phys. 08 (2018) 139.

[60] Since the soft gluon theorem is related to an infinite dimensional Kac-Moody symmetry [89] on the celestial sphere (obtained by a stereographic projection of transverse coordinates) at null infinity, these symmetries may help identify the correct boundary conditions. Note that this theorem is associated with a color memory [90]. In the Regge limit, the latter is precisely the color matrix in Eq. (4) [91] at $x^{-}= \pm \infty$.

[61] $\boldsymbol{p}_{J \perp, K \perp}$ are the transverse momenta carried by the two jets, and $z_{J}, z_{K}$ are their respective momentum fractions relative to the projectile momentum. $\gamma_{E}$ is the Euler-Mascheroni constant.

[62] Note that the NLO real gluon emission computation also contains the LO results for the production cross sections for 4-jet $\gamma+q \bar{q} g$ and 3-jet $q \bar{q} g$ [92] final states at small $x$.

[63] G. A. Chirilli, B.-W. Xiao, and F. Yuan, Inclusive Hadron productions in pA collisions, Phys. Rev. D 86, 054005 (2012).

[64] G. A. Chirilli, B.-W. Xiao, and F. Yuan, One-loop Factorization for Inclusive Hadron Production in $p A$ Collisions in the Saturation Formalism, Phys. Rev. Lett. 108, 122301 (2012).

[65] A. Stasto, B.-W. Xiao, and F. Yuan, Back-to-back correlations of Di-hadrons in dAu collisions at RHIC, Phys. Lett. B 716, 430 (2012).

[66] T. Altinoluk, N. Armesto, G. Beuf, A. Kovner, and M. Lublinsky, Single-inclusive particle production in protonnucleus collisions at next-to-leading order in the hybrid formalism, Phys. Rev. D 91, 094016 (2015).

[67] B. Ducloué, E. Iancu, A. H. Mueller, G. Soyez, and D. N. Triantafyllopoulos, Non-linear evolution in QCD at highenergy beyond leading order, J. High Energy Phys. 04 (2019) 081.

[68] Z.-B. Kang, Y.-Q. Ma, and R. Venugopalan, Quarkonium production in high energy proton-nucleus collisions: CGC meets NRQCD, J. High Energy Phys. 01 (2014) 056.

[69] Y.-Q. Ma and R. Venugopalan, Comprehensive Description of $J / \Psi$ Production in Proton-Proton Collisions at Collider Energies, Phys. Rev. Lett. 113, 192301 (2014).

[70] S. Benić, K. Fukushima, O. Garcia-Montero, and R. Venugopalan, Probing gluon saturation with next-to-leading order photon production at central rapidities in protonnucleus collisions, J. High Energy Phys. 01 (2017) 115.

[71] S. Benić and K. Fukushima, Photon from the annihilation process with CGC in the $p A$ collision, Nucl. Phys. A958, 1 (2017).

[72] S. Benić, K. Fukushima, O. Garcia-Montero, and R. Venugopalan, Constraining unintegrated gluon distributions from inclusive photon production in proton-proton collisions at the LHC, Phys. Lett B 791, 11 (2019).

[73] J. P. Blaizot, F. Gelis, and R. Venugopalan, High-energy pA collisions in the color glass condensate approach. 2. Quark production, Nucl. Phys. A743, 57 (2004).

[74] F. Dominguez, C. Marquet, A. M. Stasto, and B.-W. Xiao, Universality of multiparticle production in QCD at high energies, Phys. Rev. D 87, 034007 (2013).

[75] K. Dusling, M. Mace, and R. Venugopalan, Parton model description of multiparticle azimuthal correlations in $p A$ collisions, Phys. Rev. D 97, 016014 (2018). 
[76] K. Fukushima and Y. Hidaka, General formulae for dipole Wilson line correlators with the color glass condensate, J. High Energy Phys. 11 (2017) 114.

[77] I. Balitsky, Operator expansion for high-energy scattering, Nucl. Phys. B463, 99 (1996).

[78] Y. V. Kovchegov, Small x F(2) structure function of a nucleus including multiple pomeron exchanges, Phys. Rev. D 60, 034008 (1999).

[79] E. A. Kuraev, L. N. Lipatov, and V. S. Fadin, The Pomeranchuk singularity in monabelian gauge theories, $\mathrm{Zh}$. Eksp. Teor. Fiz. 72, 377 (1977) [Sov. Phys. JETP 45, 199 (1977)].

[80] I. I. Balitsky and L. N. Lipatov, The Pomeranchuk singularity in quantum chromodynamics, Yad. Fiz. 28, 1597 (1978) [Sov. J. Nucl. Phys. 28, 822 (1978)].

[81] A. Banfi, G. Marchesini, and G. Smye, Away from jet energy flow, J. High Energy Phys. 08 (2002) 006.

[82] G. Marchesini and A. H. Mueller, BFKL dynamics in jet evolution, Phys. Lett. B 575, 37 (2003).

[83] H. Weigert, Nonglobal jet evolution at finite N(c), Nucl. Phys. B685, 321 (2004).

[84] Y. Hatta, Relating $e+e-$ annihilation to high energy scattering at weak and strong coupling, J. High Energy Phys. 11 (2008) 057.
[85] E. Avsar, Y. Hatta, and T. Matsuo, Soft gluons away from jets: Distribution and correlation, J. High Energy Phys. 06 (2009) 011.

[86] Y. Hatta and T. Ueda, Resummation of non-global logarithms at finite $N_{c}$, Nucl. Phys. B874, 808 (2013).

[87] Y. Hatta, E. Iancu, A. H. Mueller, and D. N. Triantafyllopoulos, Resumming double non-global logarithms in the evolution of a jet, J. High Energy Phys. 02 (2018) 075.

[88] D. Neill, The asymptotic form of non-global logarithms, black disc saturation, and gluonic deserts, J. High Energy Phys. 01 (2017) 109.

[89] T. He, P. Mitra, and A. Strominger, 2D Kac-Moody symmetry of 4D Yang-Mills theory, J. High Energy Phys. 10 (2016) 137.

[90] M. Pate, A.-M. Raclariu, and A. Strominger, Color Memory: A Yang-Mills Analog of Gravitational Wave Memory, Phys. Rev. Lett. 119, 261602 (2017).

[91] A. Ball, M. Pate, A.-M. Raclariu, A. Strominger, and R. Venugopalan, Measuring color memory in a color glass condensate at electron ion colliders, Ann. Phys. (Amsterdam) 407, 15 (2019).

[92] A. Ayala, M. Hentschinski, J. Jalilian-Marian, and M. E. Tejeda-Yeomans, Polarized 3 parton production in inclusive DIS at small x, Phys. Lett. B 761, 229 (2016). 